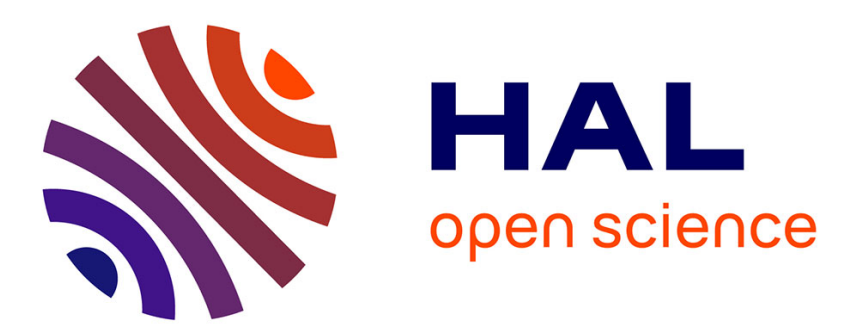

\title{
Theoretical study of xenon adsorption in UO2nanoporous matrices
}

Mehdi Colbert, Guy Treglia, Fabienne Ribeiro

\section{To cite this version:}

Mehdi Colbert, Guy Treglia, Fabienne Ribeiro. Theoretical study of xenon adsorption in UO2nanoporous matrices. Journal of Physics: Condensed Matter, 2014, 26, 10.1088/09538984/26/48/485015. hal-03040139

\section{HAL Id: hal-03040139 \\ https://hal.science/hal-03040139}

Submitted on 4 Dec 2020

HAL is a multi-disciplinary open access archive for the deposit and dissemination of scientific research documents, whether they are published or not. The documents may come from teaching and research institutions in France or abroad, or from public or private research centers.
L'archive ouverte pluridisciplinaire HAL, est destinée au dépôt et à la diffusion de documents scientifiques de niveau recherche, publiés ou non, émanant des établissements d'enseignement et de recherche français ou étrangers, des laboratoires publics ou privés. 
Theoretical study of xenon adsorption in $\mathrm{UO}_{2}$ nanoporous matrices

This content has been downloaded from IOPscience. Please scroll down to see the full text. 2014 J. Phys.: Condens. Matter 26485015

(http://iopscience.iop.org/0953-8984/26/48/485015)

View the table of contents for this issue, or go to the journal homepage for more

Download details:

IP Address: 139.124.20.101

This content was downloaded on 27/11/2014 at 12:59

Please note that terms and conditions apply. 


\title{
Theoretical study of xenon adsorption in $\mathrm{UO}_{2}$ nanoporous matrices
}

\author{
Mehdi Colbert $^{1}$, Guy Tréglia $^{2}$ and Fabienne Ribeiro ${ }^{1}$ \\ ${ }^{1}$ Institut de Radioprotection et Sûreté Nucléaire, IRSN, BP3-13115 Saint-Paul-lez-Durance, France \\ ${ }^{2}$ Centre Interdisciplinaire de Nanosciences de Marseille, CINaM, CNRS_Aix-Marseille Université, \\ Campus de Luminy, Case 913, F13288 Marseille Cedex 9, France \\ E-mail: fabienne.ribeiro@irsn.fr
}

Received 1 August 2014, revised 25 September 2014

Accepted for publication 26 September 2014

Published 12 November 2014

\begin{abstract}
We present a theoretical study of xenon incorporation in $\mathrm{UO}_{2}$ nanocavities, by means of Grand Canonical Monte Carlo calculations based on semi-empirical potentials. We first characterize the reconstruction of the matrix around an empty cavity which leads to a stoechiometry change from $\mathrm{UO}_{2}$ to $\mathrm{UO}$ in this region. Then, we determine xenon adsorption isotherms which exhibit an abrupt transition from a dilute phase to a dense one and an increase in the density of the latter phase as a function of temperature. This last result is attributed to a vibrational entropy effect by means of a mean field analysis. Finally, the pressure calculation inside the bubble proves the limitations of the usual mesoscopic models based on gas state behaviour.
\end{abstract}

Keywords: $\mathrm{UO}_{2}$, xenon, adsorption, modeling, Monte Carlo, nanoporous

(Some figures may appear in colour only in the online journal)

\section{Introduction}

Porous materials have recently attacted interest in many areas including industry, chemical engineering and material science due to their potential for use in the separation and storage of gases [1-4] and for catalysis [5]. Thus, numerous experimental as well as theoretical studies have been performed on various systems combining different nanoporous matrices and different gases. In particular, much work concerning zeolite [6-8], carbon porous matrices [9] and gas adsorption isotherms in these systems. Beyond this applied interest, such studies are also important from a fundamental point of view to characterize the effects of confinement on phase transitions such as solid/liquid or order/disorder ones [10,11].

In the nuclear field, porosities inside $\mathrm{UO}_{2}$ fuel are very important since they contribute to limit fission gas release, in particular xenon, in the gap between the fuel matrix and the cladding and then to overpressurize the latter. However, the presence of gases inside the fuel matrix impacts all its thermomechanical properties and can also increase the cladding mechanical stress through additional fuel swelling. This last phenomenon is particularly important as a safety concern since it could lead to the cladding cracking. In order to describe the effect of intragranular nanobubbles on the thermomechanical properties of $\mathrm{UO}_{2}$, micromechanical models have been developed [12,13], which need to be fed by local pressure value inside and outside the bubbles. Usually, the pressure inside the bubbles is determined using either the perfect gas or the Van der Waals equations of state. Unfortunately, these expressions are totally unsuited to the temperature and pressure ranges encountered in nuclear fuel intragranular bubbles. Indeed, experimental results indicate that the xenon pressure order of magnitude is of a few GPa in these systems $[14,15]$. That is the reason alternative equations of state have been proposed [16,17], but without taking into account the $\mathrm{Xe}-\mathrm{UO}_{2}$ interactions at the gas-matrix interface, which implies neglecting the confinement effects even though they are known to be important $[18,19]$.

The aim of this work is to go beyond these limitations by means of atomistic simulations. Indeed, computer simulations have been considered as a new research tool for a better understanding of processes occurring at the atomic scale, as well as for processes that could not be observed directly by experimental means. Here, we will first characterize the empty cavity surface reconstruction before determining the xenon adsorption isotherms into the $\mathrm{UO}_{2}$ matrix by Grand Canonical Monte Carlo (GCMC) calculations, with particular attention to the possible condensation of the gas phase. Then, we will 
calculate the pressure inside the bubble, in order to check the limits of the traditional equations of state and possibly to feed higher scale modelings. Furthermore, we will propose a mean field approach analysis of the adsorption isotherms, which could be a useful tool to enable us to go from an atomistic to a macroscopic system description.

It is worth noting that not only the methodology adopted here, but also the objectives pursued and the physics explored, are fundamentally different from those described in the article by Jelea et al [19], which can be considered as a preliminary work with respect to the current one. Indeed, its purpose was to quantify the pressure generated by a given amount of xenon introduced into a cavity in an arbitrary manner with regard to both the quantity entered (estimated from experimental data) and its crystallographic nature. On the contrary, in the approach used here, we address the problem from the other end, since our primary goal is to determine the characteristics (density and structure in particular) of a physical incorporation of xenon corresponding to given experimental conditions (temperature, chemical potential). This physical filling, characterized by the isotherms calculated here, will be the essential result of our paper, concerning both the evolution observed from GCMC calculations and the physical identification of their driving forces through their mean field analysis. In this context, the change in pressure is one of the signatures of this behaviour, the point corresponding to the paper by Jelea et al being positioned in this general (and physical) trend.

\section{Methods}

\section{1. $\mathrm{UO}_{2}$ Matrix modeling}

As previously described [20,21], the system under study is a network of perfect $\mathrm{UO}_{2}$, generated by infinitely duplicating the elementary cell in the three space directions. The $\mathrm{UO}_{2}$ elementary cell is of fluorite type and its lattice constant is $a=0.547 \mathrm{~nm}$ as determined experimentally at $296 \mathrm{~K}$ [22]. The porosities are introduced in the system as spherical cavities. These cavities are created inside cells by first eliminating all uranium and oxygen atoms contained in a sphere having a radius $R$. Then, the global stoichiometry of the system is preserved by removing excess uranium or oxygen atoms from the surface, in order to ensure the cell electroneutrality. To this aim, a criterion based on atom coordination is used, which requires determining a first neighbor distance threshold. This is achieved by the analysis of the Radial Distribution Function, which clearly separates a first coordination shell around $0.24 \mathrm{~nm}$ from the second one around $0.45 \mathrm{~nm}$. This allows us to unambiguously fix the distance threshold to the value of $0.34 \mathrm{~nm}$. Then, all the undercoordinated atoms (based on this threshold criterion) compared to the bulk reference are considered as surface atoms and are removed in priority.

In practice, we use as a starting point a perfect fluorite structure cell of 2592 atoms $(6 \times 6 \times 6$ supercells $)$, corresponding to a volume of about $35 \mathrm{~nm}^{3}$. These supercells are large enough to allow the creation of nanopores with diameters comparable to the experimental ones. We have checked that a larger $(6 \times 6 \times 12)$ cell leads to the same results which confirms the absence of non-physical boundary effects. The cavity radius $R$ is ranging from 0.4 to $1 \mathrm{~nm}$ which leads to a global porosity $f$ (ratio between the number of cancelled atoms and the total number of atom in the perfect cell) ranging from 0.05 to 0.12 .

However, the system so obtained is far from being equilibrated. Therefore, it must be relaxed by a suited thermodynamical procedure within an appropriate energetic model.

\subsection{Energetic model}

$A b$ initio methods are not suited to nanocavity modeling since it involves the study of several thousand atom systems. In addition, the physical properties of interest, such as mechanical or thermal properties, do not require a precise calculation of the electronic structure. Therefore semi-empirical potentials are well suited here.

2.2.1. UO interaction model. The system total energy $E_{\text {tot }}$ can be written analytically as a function of the atomic positions, as a sum of an electrostatic term $\left(E_{\text {Coulomb }}\right)$ and a two body interaction one $\left(E_{\text {inter }}\right)$ :

$$
E_{\text {tot }}=E_{\text {Coulomb }}+E_{\text {inter }}
$$

where $E_{\text {Coulomb }}$ is given by:

$$
E_{\text {Coulomb }}=\sum_{i} \sum_{j} \frac{q_{i} q_{j}}{4 \pi \epsilon_{0} r_{i j}}
$$

with $q_{0}=-1.2$ and $q_{U}=2.4$ the particule charges and $r_{i j}$ the interatomic distances. This term is taken into account by an usual Ewald summation.

Concerning the interaction term, there are a variety of $\mathrm{UO}_{2}$ effective energetic models in the literature [23-26]. We choose here the Basak potential [27] which accounts for the interactions involved in $\mathrm{UO}_{2}$ and correctly reproduces macroscopic quantities such as the elastic constants (see table 1, especially at high temperature and for structures far from the perfect cristal $[22,28]$. In addition, this potential reproduces fairly well surface properties and in particular surface energies for main orientations [20].

This potential is grounded on Buckingham-Hill (in the case of $\mathrm{U}-\mathrm{U}, \mathrm{O}-\mathrm{O}$ and partly $\mathrm{U}-\mathrm{O}$ ) and Morse (for the covalent part of the U-O term) interactions:

$$
E_{\text {inter }}=E_{\text {Buck }}+E_{\text {Morse }}
$$

The Buckingham-Hill component involves two terms : a short range repulsive part and an attractive Van der Waals term in $1 / r^{6}$ for $\mathrm{O}-\mathrm{O}$ :

$$
E_{\mathrm{Buck}}(r)=A \exp \left(-\frac{r}{\rho}\right)-\frac{C}{r^{6}}
$$

The Morse component has the following analytical form :

$E_{\text {Morse }}(r)=D\left[1-\exp \left(\beta\left(r^{*}-r\right)\right)\right]^{2}-1$

The different Basak potential parameters are given in table 2. 
Table 1. Comparison of elastic properties calculated using Basak potential with experimental values : $C_{i j}$ : elastic constants; $\mathrm{K}=\mathrm{Bulk}$ modulus.

\begin{tabular}{lcc}
\hline & $\begin{array}{l}\text { Basak } \\
\text { potential [27] }\end{array}$ & $\begin{array}{l}\text { Experimental } \\
\text { values [29] }\end{array}$ \\
\hline$C_{11}(\mathrm{Gpa})$ & 408.1 & 389.3 \\
$C_{12}(\mathrm{Gpa})$ & 61.2 & 118.7 \\
$C_{44}(\mathrm{Gpa})$ & 59.5 & 59.7 \\
$\mathrm{~K}(\mathrm{Gpa})$ & 176.9 & 204.0 \\
Lattice & 5.454 & 5.47 \\
parameters $(\AA)$ & & \\
\hline
\end{tabular}

Table 2. U-O Basak interaction parameters.

\begin{tabular}{llll}
\hline Parameters & $\mathrm{O}-\mathrm{O}$ & $\mathrm{U}-\mathrm{U}$ & $\mathrm{U}-\mathrm{O}$ \\
\hline $\mathrm{A}(\mathrm{eV})$ & 1633.39 & 294.745 & 693.87 \\
$\mathrm{C}\left(\mathrm{eV} \cdot \AA^{6}\right)$ & 3.950 & - & - \\
$\rho(\AA)$ & 0.327022 & 0.327022 & 0.327022 \\
$\mathrm{D}(\mathrm{eV})$ & - & - & 0.57745 \\
$\beta\left(\AA^{-1}\right)$ & - & - & 1.658 \\
$r^{*}(\AA)$ & - & - & 2.369 \\
\hline
\end{tabular}

2.2.2. Xe-UO interaction model. Concerning the interactions involving xenon atoms, we use the 'Buckingham-Hill' analytical form (equation (4)). The corresponding parameters for the interaction between xenon and matrix atoms (XeU et $\mathrm{XeO}$ ) are given by Grimes [25] and between two xenon atoms by Brearley [30]. For very short distances $\left(r<r_{\text {born }}\right)$, the Van der Waals term induces numerical divergence. New parameters ( $A, r$ and $C$ ) are then ajusted on experimental data [19]. The whole set of parameters is given in table 3 .

\subsection{Monte Carlo thermostatistic tools}

Based on the energetic model previously described, we have separated our study in two successive steps : the first one is to relax the matrix cavity in absence of xenon and the second one is to fill the so-relaxed cavity by xenon atoms. In order to check the validity of our assumption not to relax the matrix under xenon incorporation, we have in a few cases added postincorporation system relaxation studies. All the simulations are performed by Monte Carlo simulations, in canonical (NVT) ensemble for relaxation steps and in grand canonical $(\mu, \mathrm{V}, \mathrm{T})$ ensemble for the incorporation study. The choice we made to perform calculation at fixed volume is motivated by the physical situation we want to simulate $\left(\mathrm{UO}_{2}\right.$ matrix constrained by a cladding).

In the canonical ensemble, the only proposed events between a configuration $i$ with energy $E_{i}$ and a configuration $i+1$ with energy $E_{i+1}$ are the atom displacements, which are accepted with the probability $P_{i, i+1}$ :

$$
P_{i, i+1}=\mathrm{e}^{-\frac{\left(E_{i+1}-E_{i}\right)}{k T}}
$$

with $k$ the Boltzmann constant and $T$ the temperature.

In the grand canonical ensemble two other types of events are proposed, the addition or the removal of one xenon atoms, with the respective probabilities $P_{i, i+1}^{\text {add }}$ and $P_{i, i+1}^{\text {rem }}$ [31]:

$P_{i, i+1}^{\mathrm{add}}=\frac{V}{\Lambda^{3}\left(N_{i}+1\right)} \mathrm{e}^{-\beta\left[E_{i+1}-E_{i}+\mu\right]}$

$P_{i, i+1}^{\mathrm{rem}}=\frac{\Lambda^{3} N_{i}}{V} \mathrm{e}^{-\beta\left[E_{i+1}-E_{i}-\mu\right]}$

where $N_{i}$ is the number of atoms in the configuration $i, V$ the system volume, $\Lambda=\frac{h}{\sqrt{2 \pi k T m}}$, the De Broglie wavelength, $m$ the xenon atomic mass, $h$ the Plank constant and $\mu$ the fixed chemical potential.

In practice, the type of event is chosen randomly, according to the following probability: $40 \%$ for moves, $30 \%$ for additions and $30 \%$ for removals. Finally, the displacement vector is randomly chosen too, in the distance range 0 to $5 \%$ of the equilibrium $\mathrm{Xe}-\mathrm{Xe}$ distance.

The Grand Canonical Monte Carlo approach allows us to determine isothermal xenon incorporation curve, in which we plot the equilibrium xenon density $\left(\mathrm{g} \mathrm{cm}^{-3}\right)$ at a given temperature as a function of the chemical potential. The thermodynamical equilibrium is assumed to be reached when the statistical number of created particules equals that of the removed ones, which generally requires more than $3 \times 10^{8}$ proposed steps. Additional Canonical Monte Carlo steps are then achieved to average the physiscal quantities of interest (the number of particules $N_{X e}$, the xenon pressure $P$, the system energy $E \ldots$...). Typically, 10000 configurations each 3000 steps are used, to avoid correlation between consecutive steps.

\subsection{Pressure determination}

The average pressure inside the xenon bubble is obtained through the following equation, which is well suited to the case of system described by pairwise interactions and periodic boundary conditions [32]:

$\left\langle P_{\text {bulle }}\right\rangle=\left\langle\frac{N_{\mathrm{Xe}} k T}{V_{b}}\right\rangle+\left\langle\frac{1}{3 V_{b}}\left(\sum_{i}^{N_{\mathrm{Xe}}} \sum_{j \neq i}^{N} \frac{1}{2} r_{i j} f_{i j}\right)\right\rangle$

where $N_{\mathrm{Xe}}$ is the equilibrium number of xenon atoms, $N$ the total number of atoms inside the simulation box, $V_{b}$ the bubble volume (assumed spherical), $r_{i j}$ the distance between atoms $i$ and $j$ and $f_{i j}$ the interaction force between these atoms, calculated as derivative of potential energy with respect to $r_{i j}$. In this equation, the first term is the kinetical contribution (perfect gaz law term) and the second one accounts for the $\mathrm{Xe}-\mathrm{Xe}$ and the Xe-matrix interactions.

\subsection{Mean field approach}

The limitation of the previous numerical simulation is that the driving forces for the observed phenomena are hidden and then that they are hardly directly exploitable for upper scale modeling tools. To overpass this difficulty, one can simplify the description of the system, in order to be able to use a mean field approach approximation [33]. To this aim, we first decide not to take into account explicitly the atomic relaxations by using a rigid lattice description and second, to reduce the interaction models to first neighbourg pairwise (Ising-like) one. In this framework, the idea is to treat the xenon atom condensation as 
Table 3. Xe-matrix and $\mathrm{Xe}-\mathrm{Xe}$ interaction parameters.

\begin{tabular}{lllllll}
\hline Parameters & $\mathrm{XeU}$ & $\mathrm{XeXe}$ & $\mathrm{XeO}$ & $\mathrm{XeU}$ & $\mathrm{XeXe}$ & $\mathrm{XeO}$ \\
$\mathrm{r}(\AA)$ & $r<1.1$ & $r<1.5$ & $r<1.8$ & $r>1.1$ & $r>1.5$ & $r>1.8$ \\
\hline $\mathrm{A}(\mathrm{eV})$ & 2915.33 & 1171.48 & 136.7 & 6139.16 & 4934.10 & 598.00 \\
$r(\AA)$ & 0.410 & 0.466 & 0.561 & 0.34 & 0.35 & 0.42 \\
$\mathrm{C}\left(\mathrm{eV} . \AA^{6}\right)$ & 0 & 0 & 0 & 71.84 & 297.47 & 108.38 \\
\hline
\end{tabular}

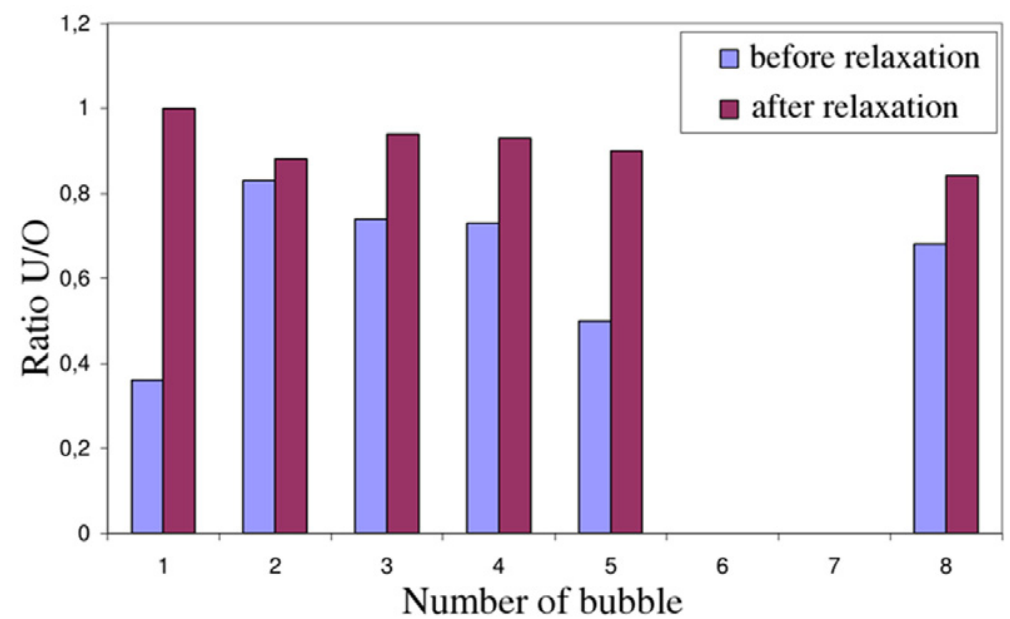

Figure 1. Bubble surface stoichiometry before and after relaxation for different initial dispersion (from 1 to 8 bubbles) corresponding to the same value of the porosity.

a binary xenon-vacancy system phase diagram determination. By this way, we will be able to determine effective rigid parwise interaction, which take into account the atomic relaxation even though projected on rigid lattice. More precisely, the Ising description of the energy system writes:

$$
E_{\mathrm{tot}}=\frac{1}{2} \sum_{n \neq m} V_{n m} p_{n} p_{m}
$$

where $p_{n}$ is the $n$ site occupation factor, which takes the value 1 if the site is occupied by a xenon atom and 0 otherwise. We choosed here to limit the range of interactions to the $Z$ first neighbours, in which case, $V_{\mathrm{nm}}=V$ if $\mathrm{n}$ and $\mathrm{m}$ are first neighbours and $V_{\mathrm{nm}}=0$ otherwise. The free energy minimisation of the previous function with respect to concentration $c=\left\langle p_{n}\right\rangle$, within the mean field approximation, leads to the usual expression:

$$
\frac{c}{1-c}=\exp \left(-\frac{\Delta F_{\mathrm{vib}}-\mu}{k T}\right)
$$

where we denote $\Delta F_{\text {vib }}$ the part of the adsorption free energy in which the configurational part has been extracted:

$$
\Delta F_{\mathrm{vib}}=c Z V-T \Delta S_{\mathrm{vib}}
$$

This equation involves two terms, the first one, $c Z V$, the interaction between impurities and $\Delta S_{\text {vib }}$ the variation of the purely vibrational entropic term with respect to concentration, i.e. related to the incorporation of one additional $\mathrm{Xe}$ atom into the system.

Through this formalism, the link between $\mu$ and $c$ as a function of the temperature is obtained trough the Monte Carlo approach, so that one can easily determine $\Delta S_{\text {vib }}$ and $Z V$ from the slope of the variation of $\Delta F_{\text {vib }}$ as a function of $c$ for different temperatures.

\section{Results}

\subsection{Cavity surface reconstruction}

The figure 1 illustrates the variation of the bubble surface stoichiometry before and after relaxation for different initial bubble dispersions corresponding to the same porosity. More precisely, we have increased the vacuum/matrix interface by sharing this total porosity in various bubble distributions (from 1 to 8 ) bubbles. As can be seen, the relaxation leads to an almost equiatomic UO composition whatever the dispersion This means that a larger number of uranium atoms are considered as surface atoms. Moreover, as can be observed in figure 2, configuration analysis indicates that oxygen atoms have been pushed far from their initial positions in the bulk towards the surface. These displacements lead to oxygen vacancy creation around the bubble and therefore to an increased number of uranium surface atoms according to our criterion. This analysis is confirmed by radial distribution functions which show that the oxygen atoms are found more numerous at a distance of approximately 0.7 to $1.1 \mathrm{~nm}$, i.e. in the surface vicinity. This confirms that the full surface relaxation requires accounting for oxygen atom mobility. This important surface relaxation is consistent with the characterisation of a surface layer presenting an effective thickness of $2.3 \mathrm{~nm}$, as previously commented in [21].

This surface thickness implies that a precise determination of the volume of the cavity, which is required to calculate the 

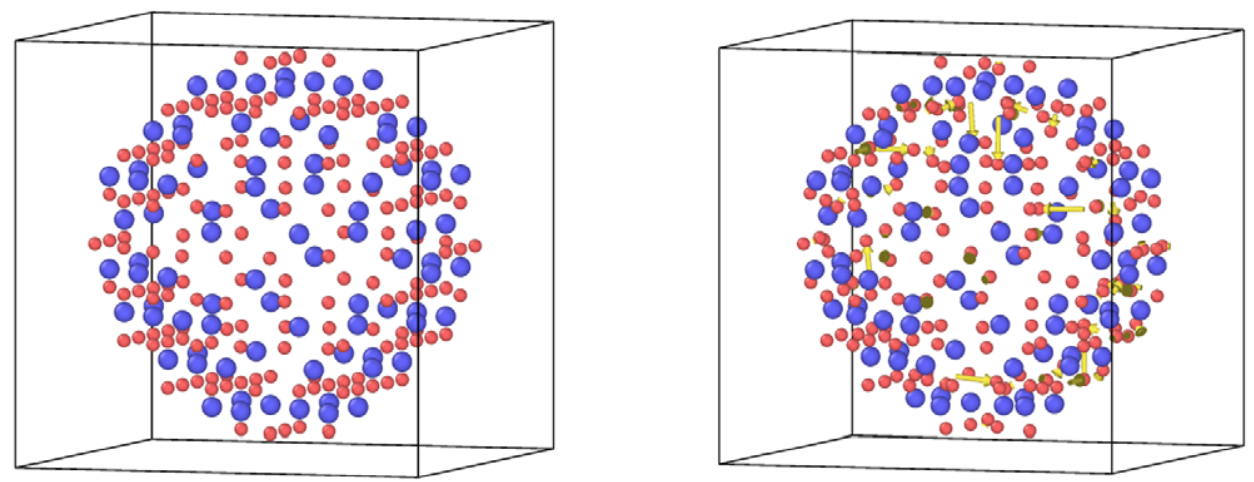

Figure 2. Periphery of the cavity before and after relaxation. Uranium atoms in blue and oxygen ones in red. The yellow arrows indicate the atom displacement under relaxation.

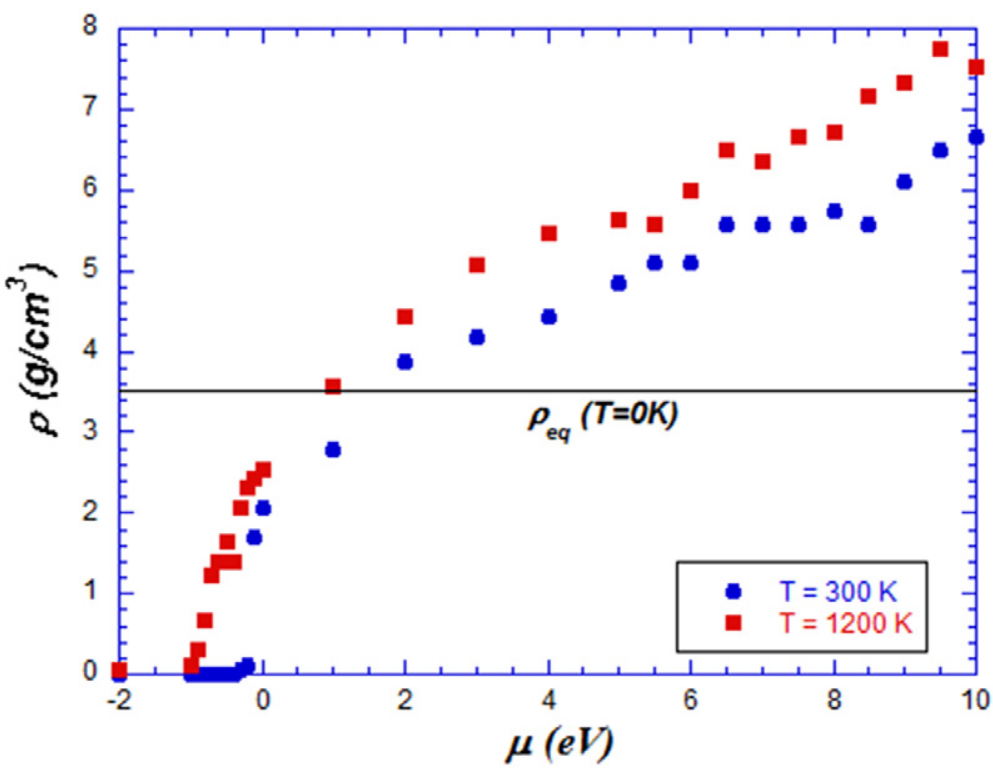

Figure 3. Evolution of the density of incorporated xenon atoms $\rho$, in $\mathrm{g} \mathrm{cm}^{-3}$ units, as a function of the chemical potential for two extreme temperatures. For the sake of comparison, the horizontal line indicates the value $\rho_{\mathrm{eq}}$ of this density for a perfect compact xenon bulk phase built with the equilibrium value of the atomic radius at $T=0 \mathrm{~K}$, namely $0.2 \mathrm{~nm}$ [36].

pressure through equation (9), by assuming a perfect spherical shape with radius $\mathrm{R}$ is somewhat approximated. In order to get an idea of the margin of error on this evaluation, we have estimated the radius of different bubbles by averaging the distances between the centre of the cavity and the atoms belonging to the thick surface layer. The margin of error on the value of the radius found within this procedure is of about $3 \%$, which means an uncertainty of about $10 \%$ in the volume determination.

\subsection{Incorporation isotherm}

As previously detailed, the physical bubble filling has been achievied within GCMC, for different chemical potentials and temperatures. The values of explored chemical potentials have been chosen in the range characteristic of the xenon state inside the fuel, namely $[-2 \mathrm{eV} ; 10 \mathrm{eV}]$, taking into account the energy of a xenon atom inside the $\mathrm{UO}_{2}$ matrix in interstitial $(\sim 20 \mathrm{eV})$ or vacancy $(\sim 5-10 \mathrm{eV})$ positions $[19,34,35]$. Under this condition, we have checked that xenon atoms only incorporate inside the cavity, as expected. The temperature range has been chosen to be consistent with the in-pile conditions and extents from $300 \mathrm{~K}$ to $1200 \mathrm{~K}$. Finally, the effect of the xenon-matrix interface is examined through different cavity radius studies between 0.4 and $1 \mathrm{~nm}$. Having not identified any significant size effect in this range, we only present in the following the results obtained for the $1 \mathrm{~nm}$ bubble radius.

Figure 3 represents the corresponding incorporation isotherms (incorporated xenon density as a function of the chemical potential for two extreme temperatures). Both curves exhibit the same general behaviour with a first zero or almost zero part followed by a more or less abrupt jump to a new plateau for approximately $\mu_{c} \simeq-0.5 \mathrm{eV}$. This behaviour is characterictic of a phase transition from dilute to condensed system, as confirmed by the microstructures shown in the figure 4. Both the values of the critical chemical potential $\mu_{c}$ and that of the saturation density $\rho_{s}$ at the plateau, depend on the temperature:

- The xenon saturation density $\rho_{s}$ corresponds to about $8 \mathrm{~g} \mathrm{~cm}^{-3}$, which is twice $\rho_{\mathrm{eq}}$ the density of a compact xenon phase with an atomic radius of $0.2 \mathrm{~nm} \mathrm{[36].}$ 


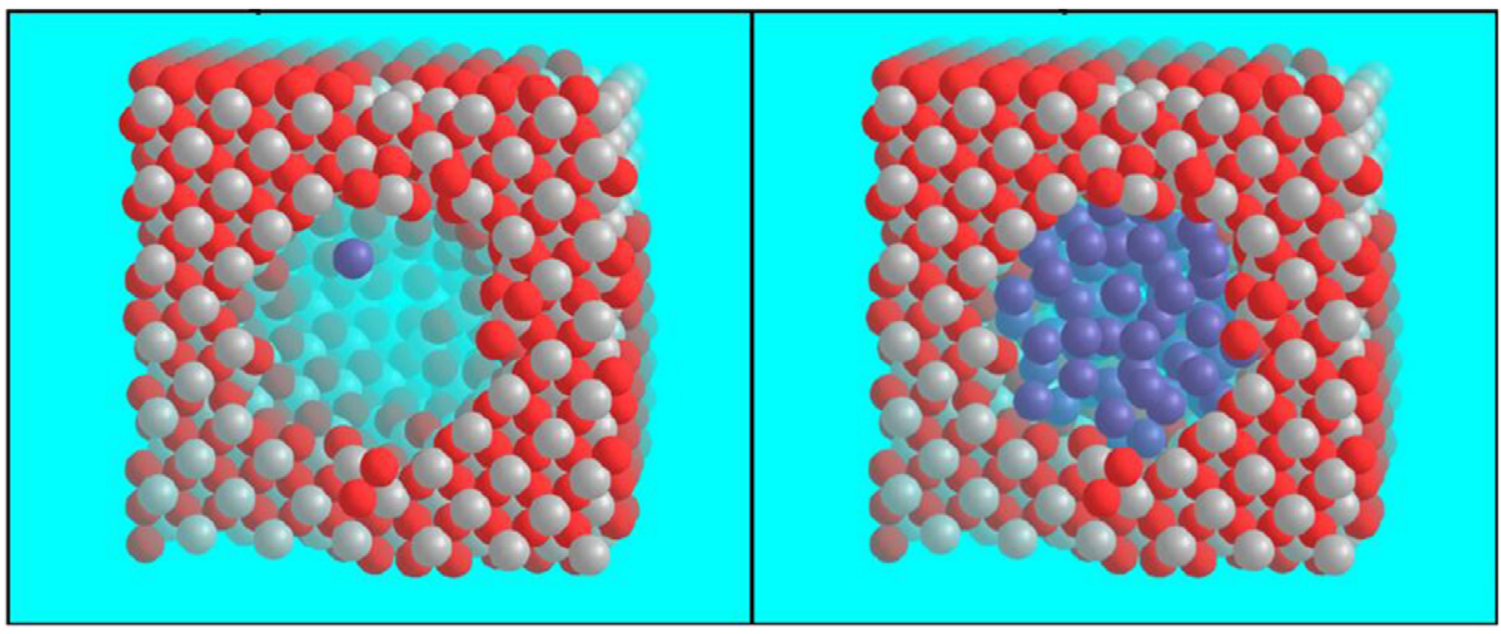

Figure 4. Microstructures observed for $\mu=-1.5 \mathrm{eV}$ and $\mu=2 \mathrm{eV}$ at $T=300 \mathrm{~K}$.

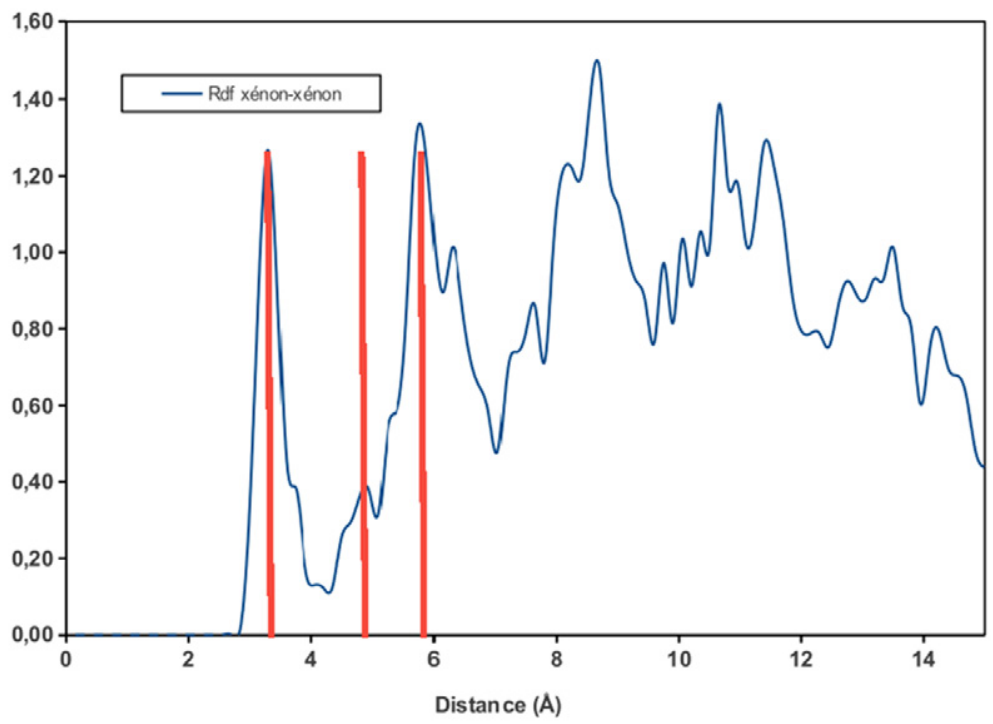

Figure 5. Radial distribution function for xenon for $\mu=2 \mathrm{eV}$ and $T=300 \mathrm{~K}$. The vertical lines stand for the first three neighbouring distances in an ideal bcc structure.

- The higher the temperature, the lower $\mu_{c}$ and the higher $\rho_{s}$. For example, $\rho_{s}$ increases by about $10 \%$ between $300 \mathrm{~K}$ and $1200 \mathrm{~K}$. Furthermore, this temperature increase smoothes the phase transition. This temperature dependence of $\rho_{s}$ value is far from being intuitive and should be related to thermal fluctuations. This point will be investigated in more details in the following section.

In order to get a microstructural signature of the phase transition from dilute to condensed phase and in particular the crystallinity or not of the later, we have plotted the radial distribution function of the xenon system. This is illustrated for $\mu=2 \mathrm{eV}$ and $T=300 \mathrm{~K}$ in figure 5 which allows to clearly identify a first neighbour shell at about $0.35 \mathrm{~nm}$, consistent with Van der Waals radius of xenon atoms. The second neighbour shell around $0.4 \mathrm{~nm}$ suggests that xenon has crystallised on a bcc lattice rather than on a fcc one. This result is consistent with high pressure and temperature xenon structuration $[37,38]$.

\subsection{Xenon bubble pressure}

Let us recall that the stress undergone inside the bubble implies a counterpart in the $\mathrm{UO}_{2}$ matrix which is a key element for nuclear safety. Therefore it is important to estimate as precisely as possible the pressure inside bubble. This point has motivated a lot of works, both experimentally $[14,15,39,40]$ and theoretically $[17,19,28]$. However, the results of these studies exhibit a large dispersion, the pressure values ranging between $1.6 \mathrm{GPa}$ and $15 \mathrm{GPa}$ at $700 \mathrm{~K}$. Therefore, one can expect that the present atomic scale study will clarify these data.

The calculation of the average pressure in the bubble is performed using the equation (9). We present in figure 6 (left) the average pressure of the bubble as a function of the chemical potential $\mu$ for two extreme temperatures. The main trends are a significant pressure increase with chemical potential, and temperature, until very high pressure values (between 25 and $45 \mathrm{GPa}$ ). Taking into account the fact that the energy of 

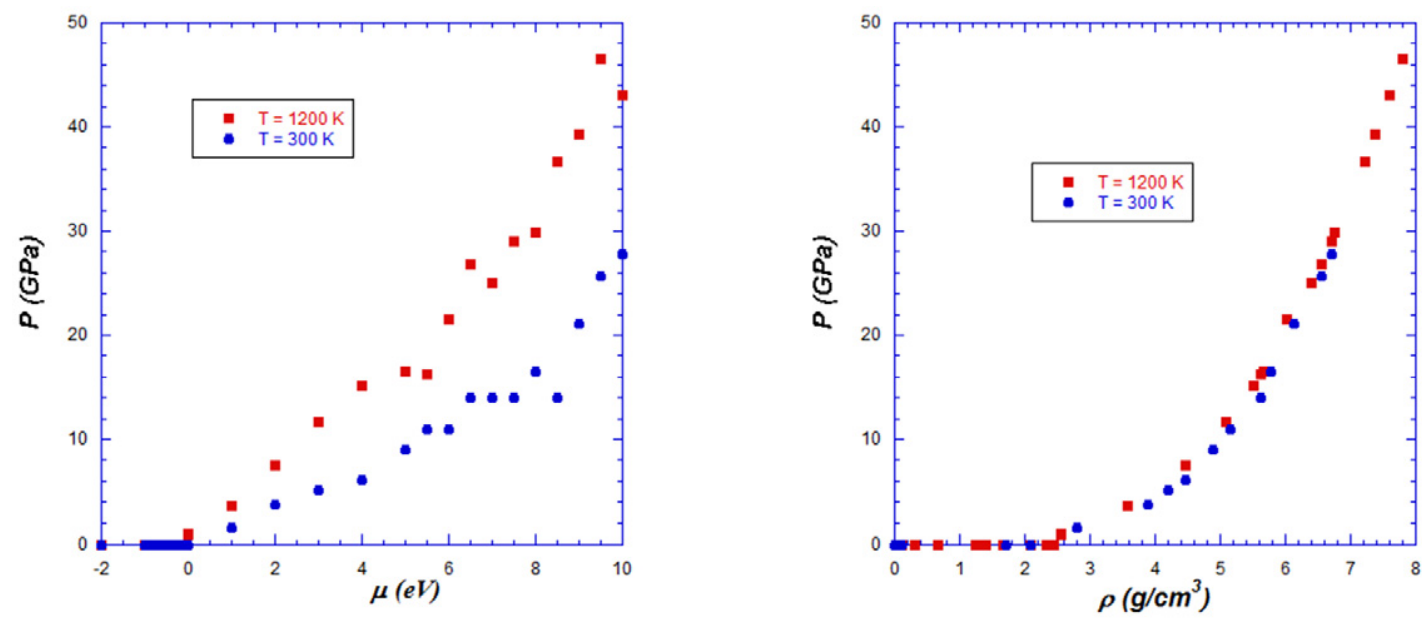

Figure 6. Total pressure (GPa) in the bubble for two extreme temperatures as a function of (left) xenon chemical potential and (right) incorporated xenon density.

incorporation of xenon in $\mathrm{UO}_{2}$ is in the range $[5 \mathrm{eV} ; 20 \mathrm{eV}]$ depending on the incorporation site, the value of the pressure obtained in the present study (between $15 \mathrm{GPa}$ and $50 \mathrm{GPa}$ ) shows that this quantity is greatly underestimated in the models based on gaseous equation of state, which are used currently. Increasing the temperature from $300 \mathrm{~K}$ to $1200 \mathrm{~K}$ enhances the pressure value of almost a factor two in the whole chemical potential range.

From the $\mu$ dependence of the xenon density (see figure 3 ) on one side and of pressure (see figure 6 left) on the other side, it is easy to extract the variation of the pressure as a function of the xenon density for the different temperatures. This is illustrated in figure 6 (right). The most striking feature is that, within this representation, the temperature effect seems to disappear.

Let us emphasize that one must be very careful to the explored thermodynamic system condition before deriving physical conclusion. Thus, following figure 6 , a temperature increase at fixed chemical potential will impose a xenon density increase inside the bubble and therefore a pressure increase, contrary to what could be erroneously deduced from an analysis at fixed density. However, it should be kept in mind that some of these findings could be slightly modified by taking into account the relaxation of the matrix during filling. In fact, the assumption of keeping the matrix rigid during the incorporation process seems to have almost no impact on this result as checked by a post incorporation global system relaxation followed by a new incorporation process, which does not lead to any noticeable matrix reorganisation or $\rho_{s}$ increase.

This absence of any significant modification of the cavity configuration can appear as somewhat contradictory with the observations made in the calculations performed by Jelea et al [19] for an a priori fixed number of Xe atoms, even though the pressure value found by these authors is consistent with the present work. In fact, the variance in the behaviours comes from the total difference in perspective already explained in the introduction. Indeed, we worked here with a fixed total cell volume ( $\mu \mathrm{VT}$ or NVT ensembles) and then verified that we do not get any change of the geometry of the cavity, whereas

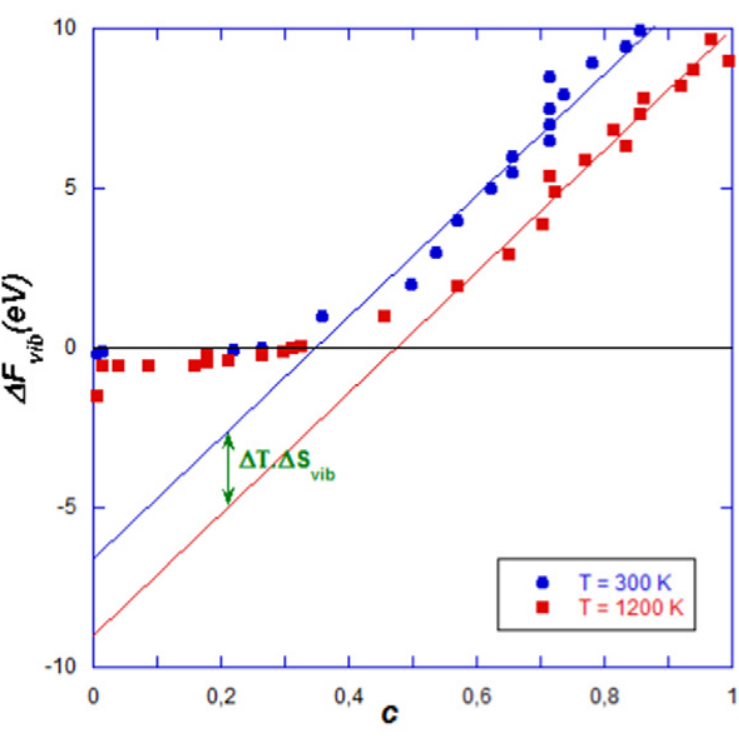

Figure 7. Free energy variation as a function of the xenon concentration for $T=300 \mathrm{~K}$ (blue circles) and $T=1200 \mathrm{k}$ (red squares).

in [19] the relaxation is performed in the NPT ensemble which is further from the physical situation and raises the problem of filling arbitrarily the cavity.

\subsection{Mean field analysis}

In order to analyze the previous results and to derive the driving forces of the isotherm behaviour, we have applied the mean field approach presented in section 2.5. The main difficulty here is the choice of the underlying rigid lattice, to be occupied by xenon atoms or vacancies, and therefore the xenon concentration definition. Here, the concentration $c$ at a given $\mu$ is defined as the ratio between the average xenon density at this potential (derived from figure 3) and $\rho_{s}=7.8 \mathrm{~g} \mathrm{~cm}^{-3}$. Using this definition and the protocole described above, we have plotted in figure 7 the variation of $\Delta F_{\text {vib }}$ as a function of $c$ for $T=300 \mathrm{~K}$ and $T=1200 \mathrm{~K}$. 
This figure raises the following comments:

- One can observe two different regimes as a function of $c$, below and above a critical concentration of $c=0.37$.

- In the first regime, $\Delta F_{\mathrm{vib}} \simeq 0$ independently of $T$ and $c$.

- In the second regime the variation of $\Delta F_{\text {vib }}$ as a function of $c$ for a given temperature is quasi linear, with a positive slope $Z V$, which is consistent with a constant value for a strongly repulsive effective pair interaction $V$, corresponding to an overstressed cristal lattice.

- The linear variation of $\Delta F_{\mathrm{vib}}$ for the second regime for different temperatures are quasi parallel, which confirms the validity of a mean field description for this system. One can then extract a value for $Z V=19 \mathrm{eV}$ from the slope of these curves and $\Delta S_{\mathrm{vib}}=32 k_{b}$ from the difference between the two curves.

- The constant value of $\Delta S_{\text {vib }}$ allows us to describe the vibrational entropy through the mean field approach : $S_{\mathrm{vib}}=c \Delta S_{\mathrm{vib}}$ which means that $\Delta S_{\mathrm{vib}}$ is the per atom entropic effective term, independent of environment.

From this analysis it appears that the vibrational entropic term is responsible of the temperature effect on the adsorption isotherm previously described.

\section{Conclusion}

We have presented here a theoretical study of xenon incorporation in $\mathrm{UO}_{2}$ nanocavities, by means of GCMC approaches based on semi-empirical potentials. The corresponding results allowed us to highlight a number of points. First, the nanostructuration of the cavity leads to a drastic change in stoechiometry at its surface. Then, xenon adsorption isotherms exhibit an abrupt transition from a dilute phase towards a dense one as a function of increasing chemical potential. The xenon density at saturation is found in good agreement with experimental values available in literature. In addition, one finds that the higher the temperature, the higher the density of xenon for a given chemical potential. In order to elucidate this behaviour, we have resorted to a mean field analysis of our system. The conclusion is that this temperature effect is related to the vibrational entropy. Complementary direct calculations of $\Delta S_{\text {vib }}$ should confirm this interpretation. This density increase with temperature leads to a pressure rise inside the bubble. Whatever the temperature, the corresponding pressure values are in agreement with most recent atomistic studies, but indicate that mesoscopic models based on gas state behaviour should be unsuited and then improved. In order to have a global physical view of the situation, the next step is now to investigate also, by similar approaches, the pressure inside the matrix. Some work is currently done in this direction.

\section{Acknowledgments}

The authors are indebted to B Legrand for his precious help, in particular for the mean field analysis. They also thank R Pellenq, A Jelea, C Garnier and M Fraczkiewicz for useful discussion and the AREVA company for its financial support.

\section{References}

[1] Morris R E and Wheatley P S 2008 Gas storage in nanoporous materials Angew. Chem. Int. Edn 47 4966-81

[2] Li J-R, Kuppler R J and Zhou H-C 2009 Selective gas adsorption and separation in metal-organic frameworks Chem. Soc. Rev. 38 1477-504

[3] Moore J D, Palmer J C, Liu Y-C, Roussel T J, Brennan J K and Gubbins K E 2010 Adsorption and diffusion of argon confined in ordered and disordered microporous carbons Appl. Surf. Sci. 256 5131-6

[4] De Jongh P E and Adelhelm P 2010 Nanosizing and nanoconfinement: new strategies towards meeting hydrogen storage goals ChemSusChem 3 1332-48

[5] Centi G and Perathoner S 2011 Creating and mastering nano-objects to design advanced catalytic materials Coord. Chem. Rev. 255 1480-98

[6] Caro J, Noack M, Kölsch P and Schäfer R 2000 Zeolite membranes-state of their development and perspective Microporous Mesoporous Mater. 38 3-24

[7] Tao Y, Kanoh H, Abrams L and Kaneko K 2006 Mesopore-modified zeolites: preparation, characterization and applications Chem. Rev. 106 896-910

[8] Keil F J, Krishna R and Coppens M 2000 Modeling of diffusion in zeolites Rev. Chem. Eng. 16 71-197

[9] Ismail A F and David L I B 2001 A review on the latest development of carbon membranes for gas separation J. Membrane Sci. 193 1-18

[10] Bonnet F, Lambert T, Cross B, Guyon L, Despetis F, Puech L and Wolf P-E 2008 Evidence for a disorder-driven phase transition in the condensation of ${ }^{4} \mathrm{He}$ in aerogels Europhys. Lett. 8256003

[11] Christenson H K 2001 Confinement effects on freezing and melting J. Phys.: Condens. Matter 13 R95-133

[12] Gatt J-M, Monerie Y, Laux D and Baron D 2005 Elastic behaviour of porous ceramics: application to nuclear fuel materials J. Nucl. Mater. 336 145-55

[13] Vincent P-G, Monerie Y and Suquet P 2009 Porous materials with two populations of voids under internal pressure: I instantaneous constitutive relations Int. J. Solids Struct. 46 480-506

[14] Martin P, Garcia P, Carlot G, Sabathier C, Valot C, Nassif V, Proux O and Hazemann J-L 2008 Xas characterisation of xenon bubbles in uranium dioxide $\mathrm{Nucl}$. Instrum. Methods Phys. Res. Sec. B: Beam Interact. Mater. At. 266 2887-91

[15] Garcia P et al 2006 A study of xenon aggregates in uranium dioxide using x-ray absorption spectroscopy $\mathrm{J}$. Nucl. Mater $352136-43$

[16] Parfitt D C and Grimes R W 2008 Predicted mechanisms for radiation enhanced helium resolution in uranium dioxide J. Nucl. Mater. 381 216-22

[17] Feng T X, Sheng L C, Zheng-He Z and Tao H G 2010 Molecular dynamics simulation of collective behaviour of $\mathrm{Xe}$ in $\mathrm{UO}_{2}$ Chin. Phys. B 1957102

[18] Alba-Simionesco C, Coasne B, Dosseh G, Dudziak G, Gubbins K E, Radhakrishnan R and MJPCM Sliwinska-Bartkowiak 2006 Effects of confinement on freezing and melting J. Phys.: Condens. Matter 18 R15

[19] Jelea A, Pellenq R J-M and Ribeiro F 2014 An atomistic modeling of the xenon bubble behaviour in the $\left\{\mathrm{UO}_{2}\right\}$ matrix J. Nucl. Mater. 444 153-60

[20] Jelea A, Colbert M, Ribeiro F, Tréglia G and Pellenq R J M 2011 An atomistic modelling of the porosity impact on $\mathrm{UO}_{2}$ matrix macroscopic properties J. Nucl. Mater. 415 210-6

[21] Colbert M, Ribeiro F and Tréglia G 2014 Atomistic study of porosity impact on phonon driven thermal conductivity: application to uranium dioxide J. Appl. Phys. 115034902 
[22] Govers K 2008 Atomic scale simulations of noble gases behaviour in uranium dioxyde $P h D$ Thesis Université Libre de Bruxelles

[23] Catlow C R A 1977 Point defect and electronic properties of uranium dioxide Proc. R. Soc. Lond. 353533

[24] Jackson R A and Catlow C R A 1985 Trapping and solution of fission $\mathrm{Xe}$ in $\mathrm{UO}_{2} \mathrm{~J}$. Nucl. Mater. 127 161-66

[25] Grimes R W and CRA Catlow 1991 The stability of fission products in uranium dioxide Phil. Trans. R. Soc. Lond. Ser. A: Phys. Eng. Sci. 335 609-34

[26] Sattonnay G and Tétot R 2013 Bulk, surface and point defect properties in $\mathrm{UO}_{2}$ from a tight-binding variable-charge model J. Phys.: Condens. Matter 25125403

[27] Basak C B, Sengupta A K and Kamath H S 2003 Classical molecular dynamics simulation of $\mathrm{UO}_{2}$ to predict thermophysical properties J. Alloys Compounds $360210-6$

[28] Geng H Y, Chen Y, Kaneta Y and Kinoshita M 2008 Molecular dynamics study on planar clustering of xenon in $\mathrm{UO}_{2}$ J. Alloys Compounds 457465

[29] Padel A and Ch De Novion 1969 Constantes elastiques des carbures, nitrures et oxydes d'uranium et de plutonium J. Nucl. Mater. 33 40-51

[30] Brearley I R and MacInnes D A 1980 An improved equation of state for inert gases at high pressures $\mathrm{J}$. Nucl. Mater. 95 239-52

[31] Leach A R 2001 Molecular Modelling: Principles and Applications (New York: Prentice Hall)
[32] Louwerse M J and Baerends E J 2006 Calculation of pressure in case of periodic boundary conditions J. Chem. Phys. Lett. 421 138-41

[33] Briki M 2013 Etude du couplage entre structure et ordre chimique dans les agrégats bimétalliques : vers l'établissement de diagrammes de phases á l'échelle nanométrique $P h D$ thesis Paris XI

[34] Nicoll S, Matzke H J and Catlow C R A 1995 A computational study of the effect of Xe concentration on the behaviour of single $\mathrm{Xe}$ atoms in $\mathrm{UO}_{2} \mathrm{~J}$. Nucl. Mater. 226 51-7

[35] Govers K, Lemehov S E and Verwerft M 2010 On the solution and migration of single $\mathrm{Xe}$ atoms in uranium dioxide? an interatomic potentials study J. Nucl. Mater. 405 252-60

[36] Kittel C 1972 Introduction à la Physique de l'état Solide (Paris: Dunod)

[37] Belonoshko A B, Ahuja R and Johansson B 2001 Molecular dynamics study of melting and fcc-bcc transitions in Xe Phys. Rev. Lett. 87165505

[38] Desgranges C and Delhommelle J 2008 Crystallization mechanisms for supercooled liquid Xe at high pressure and temperature: hybrid Monte Carlo molecular simulations Phys. Rev. B 77054201

[39] Nogita K and Une K 1997 High resolution tem of high burnup $\mathrm{UO}_{2}$ fuel J. Nucl. Mater. $250244-9$

[40] Nogita K and Une K 1998 High resolution tem observation and density estimation of Xe bubbles in high burnup $\mathrm{UO}_{2}$ fuels Nucl. Instrum. Methods Phys. Res. Sect. B: Beam Interact. Mater. At. 141 481-6 